\title{
ON THE MARX CONJECTURE FOR THE CONVEX HULLS OF FAMILIES OF STARLIKE AND CONVEX MAPPINGS
}

\author{
DAVID J. HALLENBECK
}

\begin{abstract}
We prove a Marx conjecture for the closed convex hull of the family of functions which are starlike of order $\alpha$ and $k$-fold symmetric. We obtain precise results for the functions which are starlike, starlike of order $\frac{1}{2}$, and starlike with 2-fold symmetry in their power series expansions.
\end{abstract}

Introduction. Let $\Delta$ denote the unit disk $\{z:|z|<1\}$ and let $A$ denote the set of functions analytic in $\Delta$. When $A$ is given the topology of uniform convergence on compact subsets of $\Delta$ it is known $[9, \mathrm{p} .150]$ to be a locally convex linear topological space. We recall the definition of subordination between two functions $f$ and $g$ analytic in $\Delta$. We say $f$ is subordinate to $g$, denoted $f<g$, if there exists an analytic function $\phi(z)$ so that $\phi(0)=0,|\phi(z)|<1$, and $f(z)=g(\phi(z))$ for $z$ in $\Delta$. We let $X$ denote the unit circle $\{x:|x|=1\}$ and $\mathscr{P}$ denote the set of probability measures on $X$.

We consider the class of functions denoted by $\mathrm{St}_{k}(\alpha)$ which are $k$-fold symmetric and starlike of order $\alpha$. We recall that $f(z)$ is in $\mathrm{St}_{k}(\alpha)$ if and only if

$$
f(z)=\sum_{m=0}^{\infty} a_{m k+1} z^{m k+1} \text { and } \operatorname{Re} \frac{z f^{\prime}(z)}{f(z)}>\alpha
$$

where $\alpha<1, k=1,2, \cdots$, and $z$ is in $\Delta$. The functions $\operatorname{St}_{1}(\alpha)$ were introduced in [7] by M. S. Robertson.

In [1] L. Brickman, D. J. Hallenbeck, T. H. MacGregor and D. R. Wilken determined the closed convex hull of $\mathrm{St}_{k}(\alpha)$ denoted by $\mathscr{H} \mathrm{St}_{k}(\alpha)$ to be the set of functions

$$
\left\{f: f(z)=\int_{X} \frac{z}{\left(1-x z^{k}\right)^{(2-2 x) / k}} d \mu(x) \text { and } \mu \in \mathscr{P}\right\} .
$$

In 1932 in $\left[4\right.$, p. 66] $\mathrm{A}$. Marx conjectured that if $f \in \mathrm{St}_{\mathbf{1}}(0)$ then the range of $f^{\prime}(z) \subset$ range of $\left(z /(1-z)^{2}\right)^{\prime}$ for all $z$ in $\Delta$. In [3] J. A. Hummel

Received by the editors March 7, 1973.

AMS (MOS) subject classifications (1970). Primary 30A32.

Key words and phrases. Starlike of order $\alpha, k$-fold symmetric, convex, Marx conjecture, subordination.

(c) American Mathematical Society 1974 
proved this conjecture to be false. In [5] R. McLaughlin proved that if $f \in \mathrm{St}_{1}(\alpha)$ then there exists a radius denoted by $r(\alpha)$ so that $0<r(\alpha) \leqq 1$ and the range of $f^{\prime}(z)$ is contained in the range of $\left[z /(1-z)^{2-2 \alpha}\right]^{\prime}$ for $|z|<$ $r(\alpha)$. The numbers $r(\alpha)$ were computed as the roots of a certain polynomial.

In Theorem (1) we prove that if $f \in \mathscr{H} \mathrm{St}_{k}(\alpha)$ then there exists a radius denoted by $r(\alpha, k)$ such that $0<r(\alpha, k) \leqq 1$ and the range of $f^{\prime}(z)$ is contained in the range of $\left[z /\left(1-z^{k}\right)^{(2-2 \alpha) / k}\right]^{\prime}$ for $|z| \leqq r(\alpha, k)$. For some special cases of interest, we compute the exact value of $r(\alpha, k)$. We also consider the class of univalent convex mappings denoted by $K$.

\section{The Marx conjecture for $\mathscr{H} \mathrm{St}_{k}(\alpha)$.}

THEOREM (1). If $f \in \mathscr{H} \mathrm{St}_{k}(\alpha)$, then there exists a positive number denoted by $r(\alpha, k)$ such that the range of $f^{\prime}(z)$ lies in the range of $\left[z /\left(1-z^{k}\right)^{(2-2 \alpha) / k}\right]^{\prime}$ for $|z| \leqq r(\alpha, k)$. The result is sharp.

Proof. It was proven in [1] that when $f \in \mathscr{H} \mathrm{St}_{k}(\alpha)$ then

$$
f(z)=\int_{X} \frac{z}{\left(1-x z^{k}\right)^{(2-2 \alpha) / k}} d \mu(x)
$$

for some $\mu \in \mathscr{P}$. We see by a short computation that

$$
f^{\prime}(z)=\int_{X}\left[\frac{z}{\left(1-x z^{k}\right)^{(2-2 \alpha) / k}}\right]^{\prime} d \mu(x)=\int_{X} p^{\prime}(x z) d \mu(x)
$$

where $p(z)=z /\left(1-z^{k}\right)^{(2-2 \alpha) / k}$. We will prove that $p^{\prime}(z)$ has a positive radius of convexity denoted by $r(\alpha, k)$. The range containment will then follow, since the integral can be approximated by sums of the form

$$
\sum_{i=1}^{n} \lambda_{i}\left[\frac{z}{\left(1-x_{i} z^{k}\right)^{(2-2 \alpha) / k}}\right]^{\prime}
$$

where $\lambda_{i} \geqq 0$ and $\sum_{i=1}^{n} \lambda_{i}=1$. Since $p(z)=z+\cdots$ satisfies the normalizations $p(0)=0$ and $p^{\prime}(0)=1$, it is easy to verify that $p^{\prime}(z)$ has a positive radius of convexity which we denote by $r(\alpha, k)$.

Suppose that $|z|>r(\alpha, k)$. The radius of univalence of $p(z)$ is strictly larger than $r(\alpha, k)$. Therefore, there exist two distinct points $r e^{i \theta_{1}}$ and $r e^{i \theta_{2}}$ where $|z|=r$ so that

$$
w=\frac{1}{2}\left[\frac{r e^{i \theta_{1}}}{\left(1-x r^{k} e^{k i \theta_{1}}\right)^{(2-2 \alpha) / k}}\right]^{\prime}+\frac{1}{2}\left[\frac{r e^{i \theta_{2}}}{\left(1-x r^{k} e^{k i \theta_{2}}\right)^{(2-2 \alpha) / k}}\right]^{\prime}
$$

is not the image of $|z| \leqq r$ under $p^{\prime}(z)$. To each number $z=r e^{i \theta}$ chosen so that $|z|=r>r(\alpha, k)$ and $r$ is sufficiently close to $r(\alpha, k)$ we may choose $\mu$ to be a measure with mass $\frac{1}{2}$ at each of the points $x_{1}=e^{i\left(\theta_{1}-\theta\right)}$ and 
$x_{2}=e^{i\left(\theta_{2}-\theta\right)}$. Then we see that

$$
g(z)=\int_{X} \frac{z}{\left(1-x z^{k}\right)^{(2-2 \alpha) / k}} d \mu(x)
$$

is in $\mathscr{H} \mathrm{St}_{k}(\alpha)$ but $g^{\prime}(z)=w$ is not in the image of $|z| \leqq r$ under $p^{\prime}(z)$. Hence, the result is sharp.

REMARKS. (1) Since $\mathrm{St}_{k}(\alpha) \subset \mathscr{H} \mathrm{St}_{k}(\alpha)$, it is clear that we have proven a result for the class $\mathrm{St}_{k}(\alpha)$.

(2) A more detailed argument shows that $r(\alpha, 1) \rightarrow 1$ as $\alpha \rightarrow 1$ and $r(0, k) \rightarrow 1$ as $k \rightarrow \infty$.

(3) When $k=1$ and $\alpha=0$ it is known [8, p. 33] that the radius of convexity of $\left[z /(1-z)^{2}\right]^{\prime}$ is $2-\sqrt{ } 3$ and hence $r(0,1)=2-\sqrt{ } 3$. So we have a sharp form of the Marx conjecture for $\mathscr{H} \mathrm{St}_{1}(0)$.

(4) Theorem (3) in [1] contains the result when $\alpha=\frac{1}{2}$ and $k=1$ that $\mathscr{H} \mathrm{St}_{1}\left(\frac{1}{2}\right)$ consists exactly of the functions found in $[2$, p. 94] to be $\mathscr{H} K$. It is easy to compute that $r\left(\frac{1}{2}, 1\right)=\frac{1}{2}$. We recall that the Marx conjecture for $K\left[4\right.$, p. 62] and $\mathrm{St}_{1}\left(\frac{1}{2}\right)[6$, p. 278] is known to hold for the full unit disk.

\section{The Marx conjecture for $\mathscr{H} \mathrm{St}_{2}(0)$.}

LEMMA 1. The function $g(z)=\left(1+z^{2}\right) /\left(1-z^{2}\right)^{2}=\left[z /\left(1-z^{2}\right)\right]^{\prime}$ is convex for $|z| \leqq(4-\sqrt{ } 13)^{1 / 2}$ and bivalent in all of $\Delta$.

ProOF. Clearly, if $h(z)=(1+z) /(1-z)^{2}$ is convex and univalent for $|z| \leqq 4-\sqrt{ } 13$, then $g(z)=\left(1+z^{2}\right) /\left(1-z^{2}\right)^{2}$ will be convex and bivalent for $|z| \leqq(4-\sqrt{ } 13)^{1 / 2}$. It is an easy matter to prove directly that $h(z)$ is univalent in all of $\Delta$. We know that $h(z)$ is convex and univalent if and only if $\operatorname{Re}\left[1+z h^{\prime \prime}(z) / h^{\prime}(f)\right] \geqq 0$ since $h^{\prime}(0) \neq 0$. A calculation shows that

$$
\operatorname{Re}\left(1+\frac{z h^{\prime \prime}(z)}{h^{\prime}(z)}\right)=\operatorname{Re}\left(\frac{3+8 z+z^{2}}{(1-z)(3+z)}\right) .
$$

The last expression is positive if and only if $\operatorname{Re}\left(3+8 z+z^{2}\right)(1-\bar{z})(3+\bar{z}) \geqq 0$. Let $r=|z|$ and $x=\operatorname{Re} z=r \cos \theta$. The previous condition becomes

$$
\begin{array}{r}
\left.\operatorname{Re}\left\{9-16|z|^{2}-|z|^{4}\right)-\left(6+8|z|^{2}\right) \bar{z}-3(\bar{z})^{2}+\left(24-2|z|^{2}\right)+3 z^{2}\right\} \\
=9-16 r^{2}-. r^{4}+18 x-10 r^{2} x \geqq 0 .
\end{array}
$$

So, we must decide when $p(x, r)=9-16 r^{2}-r^{4}+18 x-10 r^{2} x$ is positive. Since $x \geqq-r$ we have

$$
\begin{aligned}
p(x, r) & \geqq p(-r, r)=9-18 r-16 r^{2}+10 r^{3}-r^{4} \\
& =(r+1)(r-3)\left(-r^{2}+8 r-3\right) \geqq 0
\end{aligned}
$$

when $r \leqq 4-\sqrt{ } 13$ the smallest positive root of $p(-r, r)$. 
THEOREM (2). If $f \in \mathscr{H} \mathrm{St}_{2}(0)$, then the range of $f^{\prime}(z)$ is contained in the range of $\left[z /\left(1-z^{2}\right)\right]^{\prime}$ for $|z| \leqq(4-\sqrt{ } 13)^{1 / 2}$. The result is sharp.

ProOf. Use Lemma 1 and proceed as in the proof of Theorem (1) where $\alpha=0$ and $k=2$.

REMARKS. This result for $\mathscr{H} \mathrm{St}_{2}(0)$ and of course $\mathrm{St}_{2}(0)$ suggests the conjecture that if $f \in \mathrm{St}_{2}(0)$ then the range of $f^{\prime}(z) \subset$ the range of $\left[z /\left(1-z^{2}\right)\right]^{\prime}$ for $z$ in $\Delta$. We note that $r(0,2)=(4-\sqrt{ } 13)^{1 / 2}$ is approximately 0.63. Recall that for $\mathscr{H} \operatorname{St}_{1}\left(\frac{1}{2}\right)$ we found $r\left(\frac{1}{2}, 1\right)=\frac{1}{2}$ while for the class $\mathrm{St}_{1}\left(\frac{1}{2}\right)$ the result held in the full disk $\Delta$.

\section{A Marx-like conjecture for $\mathscr{H} K$.}

LEMMA 2. The function $g(z)=z /(1-z)^{3}$ has a radius of convexity equal to $\frac{1}{8}(7-\sqrt{33})$ and a radius of univalence equal to $\frac{1}{2}$.

Proof. It is trivial to verify that the radius of univalence of $g(z)$ is $\frac{1}{2}$. The function $g(z)$ is convex and univalent for $|z| \leqq r$ if and only if

$$
\operatorname{Re}\left[1+\frac{z g^{\prime \prime}(z)}{g^{\prime}(z)}\right] \geqq 0
$$

since $g^{\prime}(0) \neq 0$. A short calculation shows that

$$
\operatorname{Re}\left[1+z g^{\prime \prime}(z) / g^{\prime}(z)\right]=\operatorname{Re}\left[\left(1+7 z+4 z^{2}\right) /(1-z)(1+2 z)\right] .
$$

The last expression is positive if and only if

$$
\operatorname{Re}\left(1+7 z+4 z^{2}\right)(1-\bar{z})(1+2 \bar{z}) \geqq 0 .
$$

Let $r=|z|$ and $x=\operatorname{Re} z=r \cos \theta$. The previous condition becomes

$$
\begin{aligned}
\operatorname{Re}\left(1+\left(7+4 r^{2}\right) z+4 z^{2}+(1-\right. & \left.\left.14 r^{2}\right) \bar{z}-2(\bar{z})^{2}+7 r^{2}-8 r^{4}\right) \\
& =1+\left(8-10 r^{2}\right) x+4 x^{2}+5 r^{2}-8 r^{4}
\end{aligned}
$$

Consider $p(x, r)=1+\left(8-10 r^{2}\right) x+4 x^{2}+5 r^{2}-8 r^{4}$. It is easy to verify that

$$
\partial p / \partial x=8-10 r^{2}+8 x \geqq 0 \text { for } r \leqq \frac{1}{2} \text { and } x \geqq-r .
$$

Hence, to minimize $p(x, r)$ we may set $x=-r$. We then have

$$
p(-r, r)=2(1+r)\left(r-\frac{1}{2}\right)\left(-1+7 r-4 r^{2}\right)
$$

and it is simple to show $p(-r, r) \geqq 0$ for $r \leqq \frac{1}{8}(7-\sqrt{ } 33)$.

THEOREM (3). If $f \in \mathscr{H} K$, then the range of $z f^{\prime \prime}(z)$ is contained in the range of $z(z /(1-z))^{\prime \prime}$ for $|z| \leqq \frac{1}{8}(7-\sqrt{ } 33)$. The result is sharp. 
Proof. As mentioned in remark (4) above, we know that

$$
f(z)=\int_{X} z /(1-x z) d \mu(x)
$$

for $\mu$ in $\mathscr{P}$. Hence $z f^{\prime \prime}(z)=\int_{X} 2 x z /(1-x z)^{3} d \mu(x)$. By Lemma 2 and the type of arguments made in the proof of Theorem (1) the result follows.

REMARKS. (1) This result suggests the problem of finding the largest radius $r$ such that if $f \in K$ then $z f^{\prime \prime}(z)<z(z /(1-z))^{\prime \prime}$ for $|z| \leqq r$. By the above result $r \geqq \frac{1}{8}(7-\sqrt{ } 33)$.

(2) Since $\mathscr{H} \operatorname{St}_{1}\left(\frac{1}{2}\right)=\mathscr{H} K$ as mentioned in Remark (4) above, we actually have proven Theorem (3) for the classes $K$ and $\mathrm{St}_{1}\left(\frac{1}{2}\right)$.

\section{REFERENCES}

1. L. Brickman, D. J. Hallenbeck, T. H. MacGregor and D. R. Wilken, Convex hulls and extreme points of families of starlike and convex mappings, Trans. Amer. Math. Soc. (to appear).

2. L. Brickman, T. H. MacGregor, and D. R. Wilken, Convex hulls of some classical families of univalent functions, Trans. Amer. Math. Soc. 156 (1971), 91-107. MR 43 \#494.

3. J. A. Hummel, The Marx conjecture for starlike functions, Michigan Math. J. 19 (3) (1972), 257-266.

4. A. Marx, Untersuchungen über schlichte Abbildungen, Math. Ann. 107 (1932), 40-67.

5. R. McLaughlin, On the Marx conjecture for starlike functions of order $\alpha$, Trans. Amer. Math. Soc. 142 (1969), 249-256. MR 40 \#328.

6. J. A. Pfaltzgraff, On the Marx conjecture for a class of close-to-convex functions, Michigan Math. J. 18 (1971), 275-278. MR 44 \#421.

7. M. S. Robertson, On the theory of univalent functions, Ann. of Math. (2) 37 (1936), 374-408.

8. R. M. Robinson, Univalent majorants, Trans. Amer. Math. Soc. 61 (1947), 1-35. MR 8, 370.

9. A. E. Taylor, Introduction to functional analysis, Wiley, New York; Chapman \& Hall, London, 1958. MR 20 \#5411.

Department of Mathematics, University of Delaware, Newark, Delaware 19711 